\title{
River meandering dynamics
}

\author{
Boyd F. Edwards* and Duane H. Smith \\ National Energy Technology Laboratory, 3610 Collins Ferry Road, Morgantown, West Virginia 26507-0880
}

(Received 25 May 2001; published 26 March 2002)

The Ikeda, Parker, and Sawai river meandering model is reexamined using a physical approach employing an explicit equation of motion. For periodic river shapes as seen from above, a cross-stream surface elevation gradient creates a velocity shear that is responsible for the decay of small-wavelength meander bends, whereas secondary currents in the plane perpendicular to the downstream direction are responsible for the growth of large-wavelength bends. A decay length $D=H / 2 C_{f}$ involving the river depth $H$ and the friction coefficient $C_{f}$ sets the scale for meandering, giving the downstream distance required for the fluid velocity profile to recover from changes in the channel curvature. Using this length scale and a time scale $T$, we explicitly trace the observed length scale invariance to the equations of motion, and predict similar time and velocity scale invariances. A general time-dependent nonlinear modal analysis for periodic rivers reveals that modes higher than the third mode are needed to describe upstream migration of bend apexes just before oxbow cutoff, and are important to accurate calculations of the time and sinuosity at cutoff.

DOI: 10.1103/PhysRevE.65.046303

PACS number(s): 47.20.-k, 92.40.Fb, 47.54.+r

\section{INTRODUCTION}

Rivers and streams are among the most beautiful, important, and dangerous objects in nature, supplying water, hydroelectric power, transportation, and recreation, and inspiring some of the world's most beautiful poetry. They are crucial to terrestrial geomorphology, excavating huge canyon networks and forming new lands by sediment deposition. Almost a billion tons of sediment are carried annually by the Mississippi river to the Gulf of Mexico [1], where sediment deposition over the last 6000 years has increased the area of Louisiana by about $35 \%$. To preserve the navigability of Toledo Harbor, where the Maumee river empties into Lake Erie, almost a million cubic meters of sediment are dredged annually [2]. Many sandstone reservoirs of natural gas, which are responsible for $23 \%$ of the world energy consumption (second only to oil), were formed from the sediment deposits of primeval rivers. Tremendous ongoing efforts to control rivers and to maintain their navigability are partly motivated by disasters such as the 1931 flood of China's Yellow River, which killed almost four million people, and the 1993 upper-Mississippi flood, which caused \$15 billion in property damages [1].

One of the most fascinating behaviors of rivers is their tendency to meander and rework their floodplains. Some meander bends of the lower Mississippi move $20 \mathrm{~m}$ laterally per year [1], though lateral migration rates for typical actively meandering rivers are of order $1 \mathrm{~m}$ per year or less. Even on a planar floodplain, large-wavelength departures from river linearity grow in amplitude and accordingly increase the river sinuosity $S=L / L_{0}$, defined as the ratio of the total river length $L$ to the linear distance $L_{0}$ between its endpoints. As its sinuosity increases, a river occasionally meets itself and abandons an oxbow (dark, stagnant loop in Fig. 1), thereby shortening the river and reducing its sinuosity. Meander

\footnotetext{
*Permanent address: Department of Physics, West Virginia University, Morgantown, WV 26506-6315.
}

bends also migrate downstream, leading to bend distortions (Fig. 1).

The purpose of this paper is to study the mechanisms and consequences of the meandering instability. To do so, we use a meandering model derived by Ikeda, Parker, and Sawai [3], and extended by Johannesson and Parker [4], to find the migration rate at all points along a river. This model, derived from the Reynolds equations for quasisteady turbulent flow in a shallow sinuous channel, relates the migration rate to the channel centerline curvature through a linear differential equation valid to first order in the curvature, and is appropriate when the river width is small compared with bend radii.

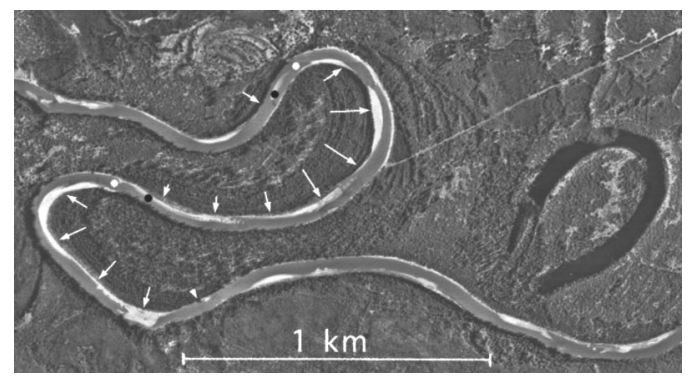

FIG. 1. Annotated aerial photograph of a reach of the Beatton River (BC7182-057, Courtesy Province of British Columbia, Canada), showing the lateral and downvalley migration of meander bends. The river flows from the left to the right sides of the photograph. The dark loop is an oxbow lake, a former river bend which was abandoned when adjacent bends in the meandering river met each other. Vegetation patterns reveal former positions of the river during the last 300 years; white arrows indicate the directions of channel shift. Meander nodes (white dots), where the channel shift is zero, are always downstream of inflection points (black dots), where the channel curvature changes sign. The distance between an inflection point and its downstream meander node is governed by the decay length $D$ [Eq. (1)], the distance required for the crossstream shear in the downstream velocity to recover from changes in the channel curvature. The separation between inflection points and meander nodes is responsible for the downstream migration of meander bends. 
Although Seminara and coworkers [5] have identified some consequences of weakly nonlinear corrections to the Ikeda, Parker, and Sawai model, this model has enjoyed a rich following [6-10] because of its ability to account quantitatively for the principal features of meandering rivers, which are the growth and downvalley migration of long-wavelength bends. To relate the migration rate to the resulting evolution of the shape of the river centerline, we employ an exact nonlinear integro-differential equation introduced by Seminara and coworkers [11,12], which correctly accounts for the essential stretching and shrinking of the evolving river. We also introduce and derive an explicit nonlinear dynamical equation [Eq. (14)] for the time-dependent river length. This equation allows the sinuosity of the river to be calculated explicitly, and serves as the key to our analytical estimates of the critical wavelength for nonlinear river meandering [13]. This wavelength separates short-wavelength bends, which decay, from long-wavelength bends, which grow.

Our physical approach yields improved understanding of the basic mechanisms of meandering. We attribute the decay of small-wavelength meander bends to the Bernoulli shear caused by the cross-stream surface elevation gradient, and trace the origin of neutral meandering stability to the competition between Bernoulli shear and the secondary flow in the plane perpendicular to the downstream direction. Critical to this competition is the decay length

$$
D=\frac{H}{2 C_{f}},
$$

which involves the river depth $H$ and the friction coefficient $C_{f}$. This decay length gives the downstream distance required for cross-stream shear in the downstream velocity to recover from changes in the channel curvature. Bernoulli shear dominates for wavelengths that are small compared with $D$, and secondary flow dominates otherwise. In studying any particular river, important insights may be gained by just knowing $D$, which sets the basic scale for the meandering wavelength as well as the distance between channel inflection points and meander nodes, where the migration rate vanishes.

We also identify the basic time scale $T$ (Sec. IV) for meandering rivers, and show that the equations of motion require only a single dimensionless parameter when scaled by $D$ and $T$. This scale invariance is responsible for the remarkable observed proportionality between meander wavelength and river width, valid over an enormous range of river widths, from $10-\mathrm{cm}$-wide laboratory flumes to the $1-\mathrm{km}$ wide Mississippi, and over remarkably different conditions including alluvial rivers, incised rivers, the gulf stream, and glacier meltwater [14]. We suggest that a similar proportionality exists between the meander period and $T$, although this proportionality is less readily observable over human lifetimes because meander periods for natural rivers are typically of the order of hundreds or thousands of years. Identification of the appropriate scales should prove useful to our eventual goal of studying the oxbow size distribution created by a meandering river $[10]$.
We present a general time-dependent nonlinear modal analysis that includes all Fourier modes describing periodic river centerlines, in contrast with previous treatments that include only a few low-lying modes $[11,12]$. We show that higher modes imply upstream migration of bend apexes in the latter stages of development of periodic rivers approaching the time of oxbow cutoff. We also calculate the precise sinuosity at cutoff. In a separate paper [15], we use level-set numerical methods to study the predictions of the Ikeda, Parker, and Sawai model for nonperiodic rivers.

Ignored herein are the effects of confining valley walls and nonuniformity in the alluvium erodibilities [9]. Many rivers such as the Beatton flow in flat flood plains, with quite uniform alluvium erodibility [16]. The "quasisteady" fluid flow through the channel is assumed to adjust quickly to the slow changes in the river shape caused by meandering. Included herein are the dependences on sinuosity of the average downstream velocity and fluid depth, which are ignored in some recent studies $[9,10,17]$. Other statistical models [18] ignore downstream migration, an essential feature of real rivers.

Section II invokes fundamental fluid physics to discuss the mechanisms of meandering. In Sec. III, we present a derivation of Seminara's evolution equation $[11,12]$, introduce our equation for the evolution of the river length, and review the Ikeda, Parker, and Sawai model [3]. In Sec. IV, these equations are written in dimensionless variables to demonstrate their scale invariance, and a series solution for the sinuosity of a sine-generated curve [19] is presented. In Sec. V, the linear stability analysis [3] of small-amplitude periodic departures from straight rivers is reexamined to further elucidate the fundamental mechanisms of meandering. In Sec. VI, we present a compact derivation of the "Kinoshita curve" describing steady finite-amplitude rivers that propagate downstream without change of form, which was first derived by Parker and Andrews [7] without the benefit of the Seminara equation. In Sec. VII, we present our general time-dependent modal analysis, and observe that this analysis precludes even-numbered modes. We also show that our explicit equation for the time-dependent river length predicts a sinuosity that agrees with numerical integration. In Sec. VIII, we study the sinuosity and bend migration near oxbow cutoff, and address the question of why our approximate nonlinear stability condition for periodic rivers should be a simple extension of the linear stability condition.

\section{FUNDAMENTAL CONSIDERATIONS}

Natural alluvial rivers continually rework their flood plains, and either degrade or aggrade these plains depending on the balance between erosion and deposition of sediment. Meandering tends to decrease the local downstream bed slope, and oxbow cutoff increases it. Local humps in the riverbed degrade faster than average because their larger fluid velocities, which are demanded by the smaller stream cross section, erode bed material more aggressively. Similarly, valleys in the riverbed aggrade faster than average because the associated smaller fluid velocities allow increased rates of deposition. These processes tend to quickly smooth 
out such humps and valleys, yielding a uniform downstream bed slope, apart from small-scale dunes and ripples [20].

The elevation of the riverbed centerline a distance $s$ measured downstream along the centerline may be written accordingly as

$$
z(s)=z_{0}-I s,
$$

where $z_{0}=z(0)$ and $z_{L}=z(L)$ are the respective centerline elevations at the upstream and downstream ends of the reach, and where $I \approx 10^{-4}-10^{-3}$ is the downstream centerline bed slope, assumed to be independent of $s$. Since all points along a reach may meander laterally with time, including the upstream and downstream ends, it is convenient to define a river reach by the elevations $z_{0}$ and $z_{L}$; the upstream and downstream ends of the reach are defined as those locations on the river with respective specified elevations $z_{0}$ and $z_{L}$. Correspondingly, the bed slope $I$, though spatially uniform, and the length $L$ of the reach measured along the river centerline must vary with time as the river meanders laterally, while the elevation drop $z_{0}-z_{L}=I L=I_{0} L_{0}$ remains constant with time, where $I_{0}$ and $L_{0}$ are the valley slope and valley length measured along a straight line between the endpoints. Accordingly, we can write a sinuosity-dependent bed slope as [3]

$$
I(S)=\frac{I_{0}}{S},
$$

where $S=L / L_{0} \approx 2-6$ is the time-dependent river sinuosity.

Natural rivers are fully developed turbulent boundary layers, with large typical Reynolds numbers $\operatorname{Re}=U H / \nu \approx 10^{6}$ involving typical average downstream velocities $U \approx 1 \mathrm{~m} / \mathrm{s}$ and depths $H \approx 1 \mathrm{~m}$, and involving the kinematic viscosity $\nu \approx 10^{-6} \mathrm{~m}^{2} / \mathrm{s}$ of water. Consequently, momentum transport is dominated by diffusion, mixing, and stretching of turbulent eddies, rather than by viscous diffusion. To effectively dissipate gravitational potential energy, aspect ratios

$$
\Gamma=\frac{2 b}{H}
$$

of river width $2 b$ to depth $H$ are typically of order $\Gamma \approx 10$ -20 . To close the system of equations, the turbulent downstream bed stress $\tau_{s}=\rho C_{f} U^{2}$ is often evaluated using a constant dimensionless friction coefficient [8] $C_{f} \approx 10^{-3}-10^{-2}$ and constant mass density $\rho$. In "quasisteady" mechanical equilibrium, the upstream bed force per unit area $\tau_{s}$ on the overlying fluid volume must balance the downstream component of gravitational force per unit area on the volume, $\rho H g I$, whence

$$
U=\left(\frac{g H I}{C_{f}}\right)^{1 / 2}
$$

The $I^{1 / 2}$ dependence of this result is a central feature of practical hydraulic equations for open channel flow [21].

Because the downstream bed slope $I$ depends on sinuosity $S$ according to Eq. (3), $U$ and $H$ must also depend on $S$ through Eq. (5). The upstream precipitation and melting con- ditions determine the river discharge $Q=2 b H U$, which is determined by the rainfall conditions and is, therefore, independent of $S$. The depth $H_{0}$ and velocity $U_{0}$ $=\left(g H_{0} I_{0} / C_{f}\right)^{1 / 2}$ of a straightened river of the same width (running in a straight line between the endpoints) must, therefore, obey $H U=H_{0} U_{0}$, whence

$$
U=U_{0} S^{-1 / 3}
$$

and

$$
H=H_{0} S^{1 / 3}
$$

for a sinuous river [3]. Increasing the sinuosity of a river, therefore, lowers its flow speed and increases its depth, and, accordingly, increases the likelihood of flooding.

The phenomenology of meandering by slow lateral migration depends crucially on cross-stream gradients of the downstream velocity. Large near-bank fluid velocities increase the local shear stress, resulting in increased bank erosion, while small near-bank velocities result in increased deposition. Rivers tend to maintain uniform widths by balancing erosion at one bank with deposition at the other. Typically, the outside "cut" bank of a meander bend erodes and the inside bank, called the "point bar," aggrades, leading to slow lateral and downstream migration of the bend. However, the roles reverse for small-radius bends resulting from oxbow cutoff. These small-radius bends are quickly straightened by high velocity and erosion near the inside bank. Thus the meandering problem reduces to finding the cross-stream velocity profiles.

Elementary fluid physics illuminates fundamental mechanisms governing these cross-stream profiles. At river bends, the water surface elevation gradient creates an outwarddirected component $\boldsymbol{\nabla} P$ of the hydrostatic pressure gradient, which supplies the centripetal body force $\mathbf{f}=-\nabla P / \rho$ necessary to accelerate fluid elements around the bend. Consequently, the downstream velocities of fluid elements entering the low-pressure region near the inside bank must increase by Bernoulli's law, whereas the downstream velocities of elements entering the high-pressure region near the outside bank must decrease [Fig. 2(a)]. This "Bernoulli shear" [22] tends to move the locus of maximum velocity toward the inside bank, and thereby straightens small-radius bends by erosion of the inside bank. Bernoulli shear also erodes the inside bank at the upstream ends of large-radius bends.

A secondary flow [22] in the plane perpendicular to the downstream direction convectively transports downstream momentum toward the outside bank and deepens the bed there. These two effects drive the locus of high velocity toward the outside bank, in direct competition with Bernoulli shear, and account for the lateral and downstream migration of large-radius meander bends. Whereas fluid elements at all depths experience the same centripetal acceleration $\mathbf{a}=\mathbf{f}$ $=-\nabla P / \rho$, downstream fluid velocities increase with increasing height above the bed, because of the vertical shear produced by the bed stress. Accordingly, low-velocity fluid elements near the bed "fall" toward the inside bank, that is, they follow circular paths whose radii $r=v^{2} / a$ are smaller than the meander bend radius, whereas high-velocity ele- 


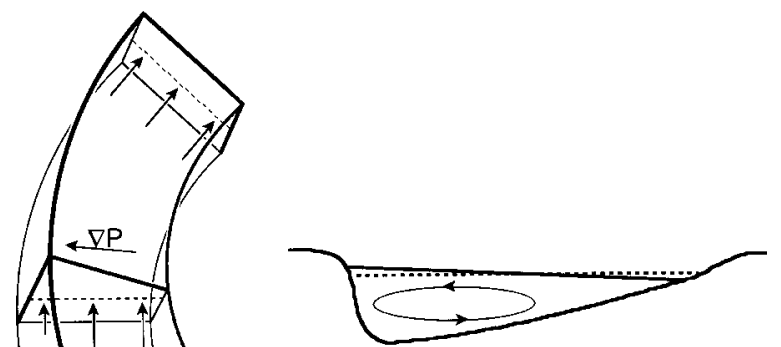

(b)

(a)

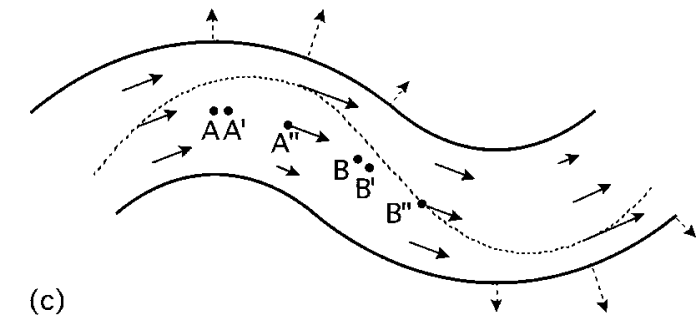

FIG. 2. Schematic velocity profiles illustrating the physical basis of meandering. (a) Three-dimensional view of a river bend to the right, showing the outward hydrostatic pressure gradient $\nabla P$ created by the surface elevation gradient, and showing the resulting Bernoulli shear in the downstream velocity (arrows). As fluid elements near the inside bank enter the low-pressure region at the bend apex, their velocities increase by Bernoulli's law. Similarly, the downstream velocities of fluid elements near the outside of the bend decrease as they approach the high-pressure region at the bend apex. This Bernoulli shear straightens small-radius bends by eroding the inside bank. (b) Vertical cross section through a river bend to the right, as seen by a river-bound observer facing downstream, showing counterclockwise secondary flow and the resulting deepening near the outside "cut" bank. This secondary flow convectively transports downstream momentum toward the outside bank, and dominates over Bernoulli shear for large-radius bends, leading to lateral and downstream migration of meander bends. (c) Schematic downstream velocities (solid arrows) for one cycle of a largewavelength sinusoidal river. Solid traces represent the river banks, whereas the dashed trace represents the locus of maximum velocity, called the thalweg, which lags behind the channel curvature by the decay length $D$ [Eq. (1)]. Large downstream velocities near a bank increase the local shear and the local bank erosion rates, leading to lateral and downstream migration of the meander pattern (dashed arrows). For typical large-radius bends such as those shown, the secondary flow overwhelms Bernoulli shear, leading to high velocities near the outsides of bends, with the largest velocities and migration rates downstream of the bend apex. For the first (right) bend, points $A, A^{\prime}$, and $A^{\prime \prime}$ respectively represent the bend apex, the location of strongest secondary flow, and the location of maximum cross-stream shear in the downstream velocity. Also at $A^{\prime \prime}$, the thalweg makes its closest approach to the left bank. Points $B, B^{\prime}$, and $B^{\prime \prime}$ respectively designate an inflection point in the channel curvature, a location of vanishing secondary flow, and a location of vanishing cross-stream shear in the downstream velocity (a meander "node"). The $A-A$ ' and $B-B$ ' distances, neglected herein, are considerably smaller than $D$, which scales the $A-A^{\prime \prime}$ and $B-B^{\prime \prime}$ distances. ments near the surface careen toward the outside bank, having radii larger than the meander bend radius. The resulting inward secondary flow near the bed scours sediment toward the inside bank, thereby deepening the river near the outside bank [Fig. 2(b)]. To avoid excessive shoaling of the flow near the inside bank, the channel then responds by shifting the bulk of the downstream flow toward the outside bank. Furthermore, the outward secondary flow near the surface convectively transports downstream momentum, which is already greatest near the surface, toward the outside bank. As seen by a river-bound observer facing downstream, bends to the right produce counterclockwise secondary flow [Fig. 2(b)] and bends to the left produce clockwise secondary flow. Because of scour, water near the outside of a bend carries much less sediment than water near the inside. Ancient Mediterranean civilizations may have recognized this fact; branch channels in their irrigation systems consistently connect to the outsides rather than the insides of bends [22].

As will be shown in Sec. III, the decay length $D$ is the downstream distance required for the cross-stream shear in the downstream velocity to recover from changes in the channel curvature. This shear decays exponentially with increasing distance along straight sections downstream of bends due to turbulent dissipation, and increases with increasing downstream distance upon entering a bend due to the secondary flow, exponentially approaching its curvaturedependent asymptotic value. These effects combine to produce a phase lag $\delta$ between the channel curvature and the fluid velocity shear profile, leading to downstream migration of meander patterns. Thus, $D \approx 100-1000 \mathrm{~m}$ governs the upstream distance over which the shape of the river contributes significantly to the local velocity profile, and supplies the basic length scale for meandering.

The secondary flow responds more quickly to changes in the channel curvature than the cross-stream shear in the downstream velocity. Decay of the secondary flow occurs over the considerably shorter length scale $D / \Gamma$ because the associated vertical shear in the cross-stream velocity is confined to the bed height $H$, whereas the cross-stream shear in the downstream velocity stretches over the entire width $2 b$ of the channel. Accordingly, the small phase lag $[8,23,24]$ between the secondary flow and the channel curvature is neglected below. The phase lag between channel curvature and secondary flow is negligible because natural river depths are an order of magnitude smaller than their widths. Figure 2(c) schematically illustrates the phase lag between the channel curvature and the cross-stream shear in the downstream velocity.

Most meandering occurs during spring and summer flooding, when river discharges, sediment loads, and bed scour are much larger than usual. In the model, however, the discharge is assumed to be constant throughout the year, and meandering is considered to occur continuously. This assumption is justified as long as the yearly migration (of order $0.5 \mathrm{~m}$ ) is small compared with the meander wavelength (of order 300 $\mathrm{m})$, and as long as the erodibility is adjusted accordingly.

\section{GOVERNING EQUATIONS}

The horizontal coordinates of the riverbed centerline may be parametrized by the distance $s$ according to $\mathbf{r}(s, t)$ 
$=[x(s, t), y(s, t)]$, which gives the position of the centerline at a distance $s$ downstream, at time $t$. Accordingly, the unit vector

$$
\hat{\mathrm{s}} \equiv \frac{\partial \mathbf{r}}{\partial s}=\hat{\mathrm{s}}(s, t)
$$

gives the horizontal projection of the downstream direction. Since $I$ is small for natural meandering rivers, the difference between $\hat{s}$ and the actual downstream direction can often be neglected. A cross-stream horizontal unit vector $\hat{\mathrm{n}}=\hat{\mathrm{n}}(s, t)$ $=\hat{\mathrm{z}} \times \hat{\mathrm{s}}$ points to the left for a riverbound observer facing downstream, where $\hat{z}$ is the unit vector pointing vertically upward. The downstream derivative

$$
\frac{\partial \hat{\mathrm{s}}}{\partial s}=-\kappa \hat{\mathrm{n}}
$$

defines the centerline curvature $\kappa=\kappa(s, t)$, measured as positive for turns to the right as seen by a riverbound observer facing downstream, and as negative for turns to the left. Also needed is the downstream derivative of the crossstream unit vector,

$$
\frac{\partial \hat{\mathrm{n}}}{\partial s}=\kappa \hat{\mathrm{s}}
$$

which follows from the derivative of $\hat{\mathrm{n}}=\hat{\mathrm{z}} \times \hat{\mathrm{s}}$. A normal velocity $v(s, t)$ measures the slow lateral migration rate of the river in the $\hat{n}$ direction, being positive for migration to the left and negative for migration to the right. [18]

Given $v(s, t)$ and $\kappa(s, t)$, the general equation of motion

$$
\frac{\partial \mathbf{r}}{\partial t}=v \hat{\mathrm{n}}+\left[u_{0}-\int_{0}^{s} \kappa\left(s^{\prime}, t\right) v\left(s^{\prime}, t\right) d s^{\prime}\right] \hat{\mathrm{s}}
$$

governs the time evolution of the river $\mathbf{r}(s, t)$. A simple geometrical derivation of this equation helps to illuminate its content: Figure 3 shows a river arc of length $s$ at time $t$, and shows the same river at time $t^{\prime}=t+d t$, after "stretching" to a new length $s^{\prime}$. Significantly, the normal displacement vector $v d t \hat{n}$ joins points on the river not generally sharing the same value of $s$. The geometric relations $d s / R=d s^{\prime} / R^{\prime}$, $R^{\prime}=R+v d t$, and $R=1 / \kappa$ involving the local radii of curvature allow us to relate the elemental arc lengths according to $d s^{\prime}=(1+\kappa v d t) d s$, whence integration yields the new river length $s^{\prime}$;

$$
s^{\prime}=s+d t \int_{0}^{s} \kappa\left(s^{\prime \prime}, t\right) v\left(s^{\prime \prime}, t\right) d s^{\prime \prime}-u_{0}(t) d t
$$

Here, the integration constant $u_{0}(t)$ is the downstream component of the migration rate $d \mathbf{r}(0, t) / d t=\partial \mathbf{r}(0, t) / \partial t$ $=v(0, t) \hat{\mathrm{n}}+u_{0}(t) \hat{\mathrm{s}}$ of the upstream end $(s=0)$ of the river.

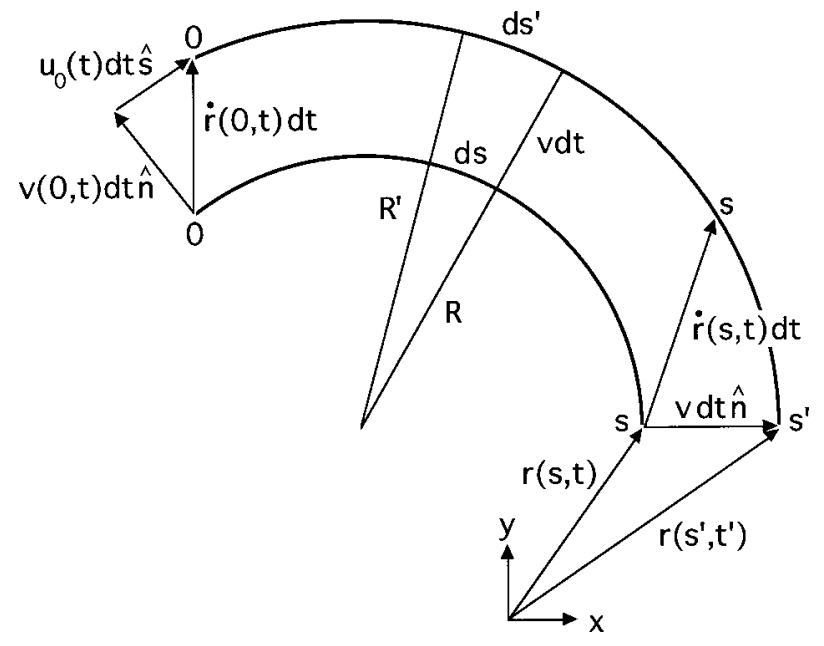

FIG. 3. River centerline arc of length $s$ at time $t$, and the same arc at a later time $t^{\prime}=t+d t$, of stretched length $s^{\prime}>s$, used in the derivation of Eq. (11). A position vector $\mathbf{r}(s, t)$ locates the downstream end of the arc at time $t$. The vector $v d t \hat{n}$ gives the displacement of this end normal to the river, where $v=v(s, t)$ is the normal velocity. Accordingly, $\mathbf{r}\left(s^{\prime}, t^{\prime}\right)$ gives the resulting position vector of the downstream end at time $t^{\prime}$. A vector $\dot{\mathbf{r}}(s, t) d t=[\partial \mathbf{r}(s, t) / \partial t] d t$ gives the displacement of the point on the river at a constant downstream distance $s$, whereas $\dot{\mathbf{r}}(0, t) d t$ gives the displacement of the $s=0$ point at the upstream end, with $\dot{\mathbf{r}}(0, t)=v(0, t) \hat{\mathrm{n}}+u_{0} \hat{\mathrm{s}}$. A wedge subtends infinitesimal arc lengths $d s$ and $d s^{\prime}$ on the old and new positions of the river, with respective local radii of curvature $R$ and $R^{\prime}$.

Equation (11) then follows simply by combining the vector relation $\mathbf{r}\left(s^{\prime}, t^{\prime}\right)=\mathbf{r}(s, t)+v d t \hat{n}$ with the first-order Taylor expansion,

$$
\mathbf{r}\left(s^{\prime}, t^{\prime}\right)=\mathbf{r}(s, t)+\left(s^{\prime}-s\right) \frac{\partial \mathbf{r}}{\partial s}(s, t)+\left(t^{\prime}-t\right) \frac{\partial \mathbf{r}}{\partial t}(s, t) .
$$

Equation (11) gives the velocity $\partial \mathbf{r} / \partial t$ of a point of constant $s$ on the river, including both normal and downstream velocity components, the latter being required by river stretching and shrinking. By setting $s=L(t)$ and $s^{\prime}=L(t+d t)$ $-u_{L}(t) d t$ in Eq. (12), we obtain a simple equation governing the time evolution of the total river length $L$;

$$
\frac{d L}{d t}=\int_{0}^{L} \kappa v d s-u_{0}+u_{L}
$$

where $u_{L}$ is the downstream component of the migration rate $d \mathbf{r}(L, t) / d t=v(L, t) \hat{\mathrm{n}}+u_{L}(t) \hat{\mathrm{s}}$ of the downstream end of the river. The downstream migration rates $u_{0}$ and $u_{L}$, which are omitted in Ref. [18], provide maximum flexibility in defining the river, and prove to be very useful below.

The downstream derivative of Eq. (11) gives a useful equation of motion governing the river angle $\theta(s, t)$, the angle between $\hat{\mathrm{s}}(s, t)$ and the fixed horizontal cartesian direction $\hat{\mathrm{x}}$, satisfying $\hat{\mathrm{s}}=\hat{\mathrm{x}} \cos \theta+\hat{\mathrm{y}} \sin \theta$ and $\hat{\mathrm{n}}=\hat{\mathrm{z}} \times \hat{\mathrm{s}}=-\hat{\mathrm{x}} \sin \theta$ $+\hat{\mathrm{y}} \cos \theta$. Accordingly, $\partial \hat{\mathrm{s}} / \partial t=\hat{\mathrm{n}} \partial \theta / \partial t$ and $\kappa=-\hat{\mathrm{n}} \cdot \partial \hat{\mathrm{s}} / \partial s$ 
$=-\partial \theta / \partial s$, and the downstream derivative of Eq. (11) reduces to a scalar equation of motion for $\theta(s, t)$;

$$
\frac{\partial \theta}{\partial t}=\frac{\partial v}{\partial s}+\kappa \int_{0}^{s} \kappa\left(s^{\prime}, t\right) v\left(s^{\prime}, t\right) d s^{\prime}-\kappa u_{0} .
$$

Seminara and co-workers previously derived this equation using a different approach $[11,12]$. Given a solution $\theta(s, t)$ of this equation, the cartesian components of $\mathbf{r}(s, t)$ may be recovered simply by integrating Eq. (8), yielding

$$
\begin{aligned}
& x(s, t)=x_{0}(t)+\int_{0}^{s} \cos \left[\theta\left(s^{\prime}, t\right)\right] d s^{\prime}, \\
& y(s, t)=y_{0}(t)+\int_{0}^{s} \sin \left[\theta\left(s^{\prime}, t\right)\right] d s^{\prime} .
\end{aligned}
$$

The case of a long river of uniform curvature $\kappa=1 / R(t)$ serves to check the nonlinear formalism and to illustrate stretching. Such a river is a vertical right-handed helix of time-dependent radius $R(t)$ [see Eq. (2)], whose flood plain is shaped like a spiral staircase. Setting $\partial \kappa / \partial s=\partial v / \partial s=0$ in the downstream derivative of Eq. (15) leaves $d \kappa / d t+\kappa^{2} v$ $=0$, which is satisfied immediately by the expected normal velocity $v(t)=d R / d t$. Furthermore, integrating Eq. (14) for a river of initial length $L(0)=R(0) \phi$ and for $u_{0}=0$ yields the expected time-dependent length $L(t)=R(t) \phi$, where $\phi / 2 \pi$ is the fixed number of helix cycles. Thus, Eqs. (14) and (15) capture the correct nonlinear dynamics of stretching for spatially uniform, time-dependent curvature.

To determine $v(s, t)$, we appeal to the celebrated model pioneered by Ikeda, Parker, and Sawai [3]. This model, which has been subsequently discussed and extended by numerous authors [4,6-10], employs the Saint Venant equations of shallow steady incompressible turbulent flow in a sinuous channel of uniform half-width $b$ to obtain a depthaveraged downstream fluid velocity of the form $u(s, n)=U$ $+u^{\prime}(s, n)$, where $U$ is the reach-averaged velocity, $u^{\prime}$ is a first-order correction due to stream curvature, and $n$ is the cross-stream coordinate measured as positive toward the left bank. To account for cross-stream shear in the downstream velocity, the normal migration rate $v(s, t)$ is taken to be proportional to the left-bank excess velocity $u^{\prime}(s, b)$ according to

$$
v(s, t)=E u^{\prime}(s, b),
$$

with a small positive dimensionless erodibility typically of order $E \approx 2 \times 10^{-8}$ [7]. Accordingly, the river migrates to the left $(v>0)$ when the left-bank velocity is higher than average, reflecting higher erosion rates, and the river migrates to the right $(v<0)$ when the left-bank velocity is lower than average, reflecting increased stagnation and sediment deposition. Since the right-bank excess velocity satisfies $u^{\prime}(s$, $-b)=-u^{\prime}(s, b)$, erosion at one bank always balances deposition at the other, thereby, allowing the river to maintain constant width. Points along the river centerline with vanishing cross-stream shear $u^{\prime}(s, b)=0$ and vanishing migration rate $v(s, t)=0$ are called meander nodes.
Including subsequent corrections [4], the Ikeda, Parker, and Sawai model [3] relates $u^{\prime}(s, b)$ and $\kappa(s, t)$ to first order according to

$$
\frac{\partial u^{\prime}(s, b)}{\partial s}+\frac{u^{\prime}(s, b)}{D}=b U\left(-\frac{\partial \kappa}{\partial s}+\frac{\mathcal{P} \kappa}{D}\right)
$$

where

$$
\mathcal{P}=\frac{F^{2}+A+A_{s}-1}{2}
$$

measures the strength of secondary flow relative to Bernoulli shear, and involves the Froude number $F=U /(g H)^{1 / 2}$, the ratio of the flow velocity to the wave velocity, and other constants to be explained shortly. The first term on the left side of Eq. (19) gives the rate of change in $u^{\prime}(s, b)$ with downstream distance. For $D=H / 2 C_{f} \rightarrow \infty$, Eq. (19) easily yields a left-bank excess velocity $u^{\prime}(s, b)=-b U \kappa$ that is $180^{\circ}$ out of phase with the centerline curvature, thereby, giving increased downstream velocities near the inside bank for both left and right turns in the river [Fig. 2(a)]. The first term on the right side of Eq. (19) accordingly represents Bernoulli shear. The second term on the left side governs the turbulent decay of cross-stream shear. The second term on the right side ignores the phase lag between the curvature and $u^{\prime}(s, b)$ [4], but includes, through $A_{s}$, the convective transport of downstream momentum (toward the outside bank) by the secondary flow. The cross-stream bed slope $A$ appearing in the bed-elevation equation $z(s, n)=z_{0}-I s-A \kappa(s) n$ [see Eq. (2)] accounts for the shift of downstream momentum toward the outside bank due to bed deepening, which is also caused by the secondary flow. For the typical values [7] $\mathcal{P}$ $\approx 5$ and $F \approx 0.4, A$ and $A_{s}$ dominate in the second term on the right side of Eq. (19), combining to represent the overall effect of secondary flow on the cross-stream shear. We treat $A$ as a constant, ignoring its insignificant $S$-dependence $A$ $\sim F H \sim S^{-1 / 6}[25,26]$. Lacking any information about the $S$ dependence of $A_{s}$, we also treat it as a constant. Finally, we ignore the 1-2\% correction supplied by $F$, and, therefore, treat $\mathcal{P}$ as a pure dimensionless constant [7], here dubbed the "Parker number" in honor of Gary Parker's many contributions to the field. Some previous studies $[9,10,17]$ ignore the sinuosity dependences of $U$ and $D$ in Eq. (19), which are crucial to the conclusions reached in Secs. VII and VIII.

To determine the distance required for the cross-stream shear to recover from changes in the channel curvature, we write the solution to Eq. (19) for the simple case of uniform $\kappa$,

$$
u^{\prime}(s, b)=b U \mathcal{P} \kappa+\left[u^{\prime}(0, b)-b U \mathcal{P} \kappa\right] e^{-s / D} .
$$

Here, $u^{\prime}(0, b)$ is the value of $u^{\prime}(s, b)$ at the upstream end of the reach, and $b U \mathcal{P}_{\kappa}$ is the value approached asymptotically with increasing distance downstream, with decay constant $D$. Accordingly, $D$ gives the distance required for the crossstream shear to recover from changes in $\kappa$, as claimed in Sec. II. When the cross-stream shear $u^{\prime}(s, b)$ does not match the value $b U \mathcal{P} \kappa$ prescribed by the local curvature, turbulence 
drives it toward this value exponentially with increasing distance downstream. Note that setting $\partial \kappa / \partial s=0$ ignores Bernoulli shear entirely.

\section{SCALE INVARIANCE AND DIMENSIONLESS VARIABLES}

Combining Eqs. (18) and (19) allows us to relate $v(s, t)$ and $\kappa(s, t)$ according to

$$
\frac{\partial v}{\partial s}+\frac{v}{D}=b E U\left(-\frac{\partial \kappa}{\partial s}+\frac{\mathcal{P} \kappa}{D}\right) .
$$

This meandering equation possesses natural sinuositydependent length and time scales,

$$
D=\frac{H}{2 C_{f}}=D_{0} S^{1 / 3}
$$

and

$$
T=\frac{D^{2}}{b E U}=T_{0} S,
$$

where $D_{0}=H_{0} / 2 C_{f}$ and $T_{0}=D_{0}^{2} / b E U_{0}$ are the corresponding scales for a straightened river [Eqs. (6) and (7)]. These sinuosity-dependent scales, therefore, govern the length and time scales for meandering, and account for the remarkable observed meander wavelength scaling of natural meandering rivers [14]. Furthermore, we predict that the meandering period of natural rivers should scale as $T$, and that the downstream migration rate should scale as $D / T$. These scale invariances are less obvious to observe in natural rivers because of the long typical time scales $T$ associated with meandering, which are of order hundreds or thousands of years. On the other hand, since $D$ is typically of the order of hundreds or thousands of meters, the length scale invariance is easily observed in maps or aerial photographs.

Although $D$ and $T$ set the natural length and time scales for the problem, they are inconvenient as scales for dimensionless variables because they depend on time through $S$. Accordingly, we employ the time-independent length and time scales $D_{0}$ and $T_{0}$ for a straightened river to define dimensionless variables according to $s=D_{0} \tilde{s}, \quad t=T_{0} \tilde{t}$, $L(t)=D_{0} \widetilde{L}(\tilde{t}), \quad L_{0}=D_{0} \widetilde{L}_{0}, \quad \mathbf{r}(s, t)=D_{0} \tilde{\mathbf{r}}(\tilde{s}, \tilde{t}), \quad v(s, t)$ $=D_{0} T_{0}^{-1} \tilde{v}(\tilde{s}, \tilde{t}), \quad u_{0}(t)=D_{0} T_{0}^{-1} \tilde{u}_{0}(\tilde{t}), \quad$ and $\quad \kappa(s, t)$ $=D_{0}^{-1} \tilde{\kappa}(\tilde{s}, \tilde{t})$. These allow us to rewrite Eqs. (14), (15), and (22) as

$$
\begin{gathered}
\frac{\partial \theta}{\partial \tilde{t}}=\frac{\partial \tilde{v}}{\partial \tilde{s}}+\tilde{\kappa} \int_{0}^{\tilde{s}} \tilde{\kappa}\left(\tilde{s}^{\prime}, \tilde{t}\right) \tilde{v}\left(\tilde{s}^{\prime}, \tilde{t}\right) d \tilde{s}^{\prime}-\tilde{\kappa} \tilde{u}_{0}, \\
\frac{d \tilde{L}}{d \tilde{t}}=\int_{0}^{\tilde{L}} \tilde{\kappa} \tilde{v} d \tilde{s}-\tilde{u}_{0}+\tilde{u}_{L}, \\
S^{1 / 3} \frac{\partial \tilde{v}}{\partial \tilde{s}}+\tilde{v}=-\frac{\partial \tilde{\kappa}}{\partial \tilde{s}}+\frac{\mathcal{P}}{S^{1 / 3}} \tilde{\kappa},
\end{gathered}
$$

with

$$
\tilde{\kappa}=-\partial \theta / \partial \tilde{s}
$$

and a lone dimensionless parameter $\mathcal{P}$. Dropping the tildes in Eq. (25c) gives Eq. (1) of Ref. [13], apart from a misplaced minus sign in the latter equation. Equations (16) and (17) reduce to

$$
\begin{aligned}
& \tilde{x}(\tilde{s}, \tilde{t})=\tilde{x}_{0}(\tilde{t})+\int_{0}^{\tilde{s}} \cos \theta\left(\tilde{s}^{\prime}, \tilde{t}\right) d \tilde{s}^{\prime}, \\
& \tilde{y}(\tilde{s}, \tilde{t})=\tilde{y}_{0}(\tilde{t})+\int_{0}^{\tilde{s}} \sin \theta\left(\tilde{s}^{\prime}, \tilde{t}\right) d \tilde{s}^{\prime} .
\end{aligned}
$$

Equation (25b) governs the time evolution of the river sinuosity $S=\widetilde{L} / \widetilde{L}_{0}$. For flood plains sloping in the $x$ direction only, the sinuosity may alternatively be computed directly from Eq. (26a) by writing $\widetilde{L}_{0}=\tilde{x}(\widetilde{L}, \tilde{t})-\tilde{x}(0, \tilde{t})$, whence

$$
\frac{1}{S}=\frac{1}{\tilde{L}} \int_{0}^{\tilde{L}} \cos \theta(\tilde{s}, \tilde{t}) d \tilde{s}
$$

The scaled equations (25) governing the time evolution of meandering rivers contain nonlinear integral terms [in Eqs. (25a) and (25b)] associated with stretching and shrinking, and are otherwise linear.

The sinuosity $S$ of the ubiquitous "sine-generated curve" [19] $\theta(\tilde{s})=\epsilon \sin q \tilde{s}$ can be determined analytically, for arbitrary amplitudes $\epsilon$ and centerline wave numbers $q=2 \pi / \tilde{L}$, by inserting $\cos \theta=\sum_{l=0}^{\infty}(-1)^{l} \theta^{2 l} /(2 l)$ ! into Eq. (27). The result is

$$
\frac{1}{S}=\sum_{l=0}^{\infty} \frac{(-1)^{l}(2 l-1) ! !}{2^{l} l !(2 l) !} \epsilon^{2 l},
$$

where $(2 l-1) ! !=(2 l-1)(2 l-3) \cdots 3 \cdot 1$, and $(-1) ! !=1$. Retaining only the first four terms

$$
\frac{1}{S}=1-\frac{\epsilon^{2}}{4}+\frac{\epsilon^{4}}{64}-\frac{\epsilon^{6}}{2304}
$$

is sufficient to give $S$ to within $2 \%$ for $S \leqslant 7$ (Fig. 4).

\section{LINEAR STABILITY ANALYSIS}

The linear stability analysis of small-amplitude sinusoidal rivers [3] further validates the general equation of motion for the river angle, Eq. (25a). We consider small-amplitude traveling-wave perturbations about a straight river of the form

$$
\begin{aligned}
& \theta(\tilde{s}, \tilde{t})=\operatorname{Re}\left[\epsilon e^{i(q \tilde{s}-\Omega \tilde{t})}\right], \\
& \tilde{v}(\tilde{s}, \tilde{t})=\operatorname{Re}\left[\eta e^{i(q \tilde{s}-\Omega \tilde{t})}\right] .
\end{aligned}
$$




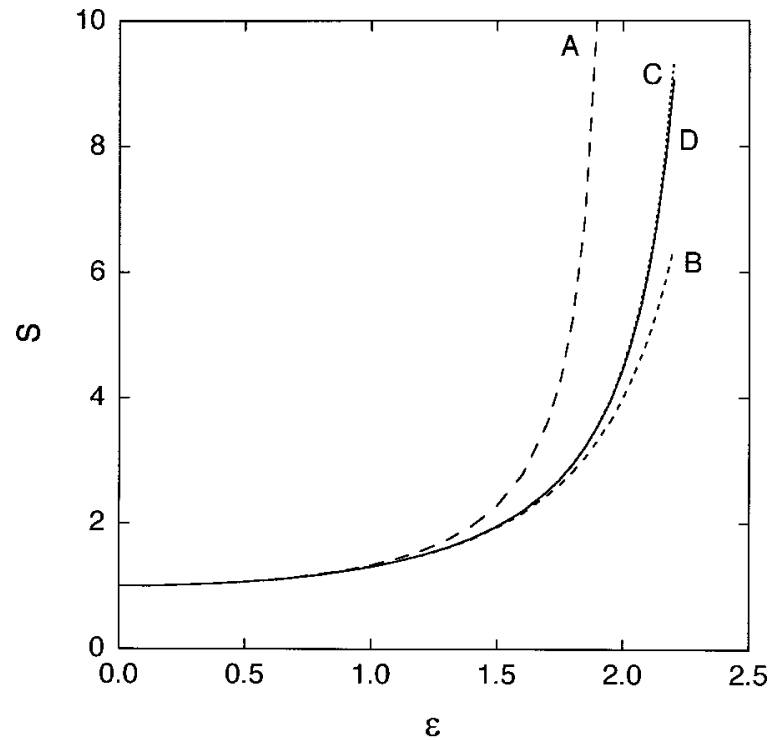

FIG. 4. Successive explicit series approximations of the sinuosity $S$ of the sine-generated curve as a function of the amplitude $\epsilon$, according to Eq. (29). Traces A, B, and C respectively include terms through order $\epsilon^{2}, \epsilon^{4}$, and $\epsilon^{6}$. Trace D gives the exact sinuosity. Traces $\mathrm{C}$ and D differ by at most $2 \%$ for $S \leqslant 7$.

To first order in the amplitudes $\epsilon$ and $\eta$, Eq. (26a) gives $\tilde{x}$ $=\tilde{s}$, whence $\widetilde{L}=\widetilde{L}_{0}=2 \pi / q$ and $S=1$. Accordingly, taking $\epsilon$ to be real, Eqs. (25a), (25c), and (26b) give, to first order,

$$
\begin{gathered}
\theta(\tilde{s}, \tilde{t})=\epsilon e^{\sigma \tilde{t}} \cos (q \tilde{s}-\omega \tilde{t}), \\
\tilde{\kappa}(\tilde{s}, \tilde{t})=q \epsilon e^{\sigma \tilde{t}} \sin (q \tilde{s}-\omega \tilde{t}), \\
\tilde{y}(\tilde{s}, \tilde{t})=q^{-1} \epsilon e^{\sigma \tilde{t}} \sin (q \tilde{s}-\omega \tilde{t}), \\
\tilde{v}(\tilde{s}, \tilde{t})=q^{-1}|\Omega| \epsilon e^{\sigma \tilde{t}} \sin (q \tilde{s}-\omega \tilde{t}-\delta),
\end{gathered}
$$

with dimensionless wave number $q$, frequency $\omega=\operatorname{Re} \Omega$, growth rate $\sigma=\operatorname{Im} \Omega$, phase velocity $c=\omega / q$, and phase lag $\delta$ satisfying

$$
\begin{aligned}
& \omega=\frac{1+\mathcal{P}}{1+q^{2}} q^{3}, \\
& \sigma=\frac{\mathcal{P}-q^{2}}{1+q^{2}} q^{2}, \\
& c=\frac{1+\mathcal{P}}{1+q^{2}} q^{2}, \\
& \delta=\tan ^{-1}\left(\frac{\omega}{\sigma}\right) .
\end{aligned}
$$

Equations (32a) and (32b) correspond to Eq. (18) of Ref. [3].

The growth rate $\sigma$ is positive for $q<q_{c}=\mathcal{P}^{1 / 2}$, and reaches its maximum value $\sigma_{m}=2+\mathcal{P}-2(1+\mathcal{P})^{1 / 2}$ where $q^{2}=q_{m}^{2}=-1+(1+\mathcal{P})^{1 / 2}($ Fig. 5), with $\mathcal{P}$ of order $1-10$. For

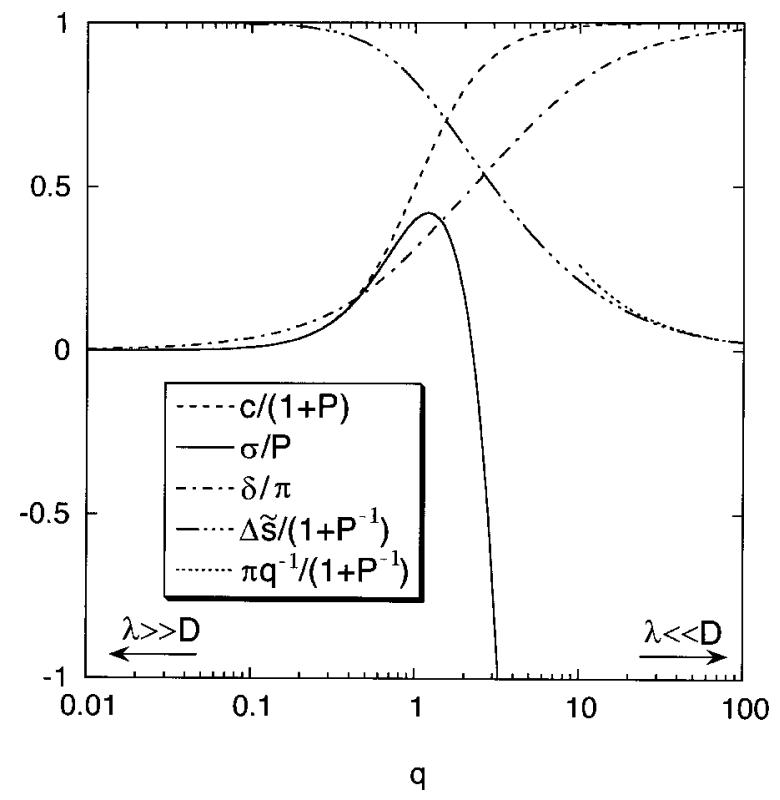

FIG. 5. Dimensionless growth rate $\sigma$, phase velocity $c=\omega / q$, nodal phase lag $\delta$, nodal displacement $\Delta \tilde{s}=\delta / q$ and the $q \rightarrow \infty$ limit $\Delta \tilde{s} \rightarrow \pi / q$ for small-amplitude sinuosoidal perturbations of dimensionless wave number $q$ about a straight river, according to Eqs. (31d) and (32). Although the typical value $\mathcal{P}=5$ is used for the plot, the $q \rightarrow 0$ and $q \rightarrow \infty$ limits of the scaled parameters $\sigma / \mathcal{P}, c /(1$ $+\mathcal{P}), \delta / \pi$, and $\Delta \tilde{s} /\left(1+P^{-1}\right)$ are independent of $\mathcal{P}$, hence plots for other values of $\mathcal{P}$ differ only in the details near $q=1$.

the typical Beatton River value $\mathcal{P}=5$ (Ref. [7]), we obtain $q_{c}=2.24, q_{m}=1.20, \sigma_{m}=2.10, \omega_{m}=4.27, \delta_{m}=64^{\circ}$, and $c_{m}=\omega_{m} / q_{m}=3.55$. Since these numbers are of order one, the length and time scales $D$ and $T$ capture the correct scales for the problem. Thus, the critical wavelength $\lambda_{c}$ $=2 \pi D_{0} / q_{c}$ in conventional units divides the longwavelength regime, for which meander bends grow in amplitude, from the short-wavelength regime, for which bends decay. Figure 5 also shows how $c$, the downstream migration rate of meander bends, approaches the maximum value 1 $+\mathcal{P}$ for short wavelengths and vanishes for long wavelengths. The phase lag $\delta_{m}=64^{\circ}$ between the channel curvature and the downstream velocity shear profile greatly exceeds the estimates $13^{\circ}[23]$ and $24^{\circ}$ [8] of the average phase lag between the channel curvature and the secondary flow in natural rivers; this latter phase lag is ignored herein.

Of particular interest is the nodal phase lag $\delta$ between river inflection points such as point $B$ in Fig. 2(c), where $\tilde{y}(\tilde{s}, \tilde{t})=\tilde{\kappa}(\tilde{s}, \tilde{t})=0$, and velocity nodes such as point $B^{\prime \prime}$, where the lateral migration velocity $\tilde{v}(\tilde{s}, \tilde{t})$ vanishes. This phase lag vanishes for long wavelengths $\lambda$, indicating that very long-wavelength meander bends grow in amplitude with little accompanying downstream migration. As $\delta$ grows with decreasing wavelength, more and more downstream migration accompanies the lateral growth. The phase lag reaches $\delta=\pi / 2$ at the critical wavelength $\lambda=\lambda_{c}$, and approaches $\delta=\pi$ for short wavelengths, where $\tilde{y}(\tilde{s}, \tilde{t})$ and $\tilde{v}(\tilde{s}, \tilde{t})$ are completely out of phase, accounting for the rapid straightening of small-wavelength bends, due to Bernoulli 
shear. For wavelengths $\lambda>\lambda_{c}$ relevant to river meandering (all others quickly disappear), the decay length $D$ sets the scale for the downstream distance between inflection points and velocity nodes, called the nodal displacement $\Delta \tilde{s}=\delta / q$ (Fig. 5).

\section{STEADY PERIODIC FINITE-AMPLITUDE SOLUTIONS}

Steady periodic finite-amplitude solutions that migrate downstream at a constant speed without changing form follow directly from Eqs. (25a) and (25c), without resorting to an intermediate bend equation (Eq. (11) in Ref. [27]), by simply demanding that each point of fixed $s$ on the river travel in the $\hat{\mathrm{x}}=\hat{\mathrm{s}} \cos \theta-\hat{\mathrm{n}} \sin \theta$ direction with constant dimensionless speed $c$ according to

$$
\frac{\partial \tilde{\mathbf{r}}}{\partial \tilde{t}}=c \hat{\mathrm{x}}=\tilde{u} \hat{\mathrm{s}}+\tilde{v} \hat{\mathrm{n}}
$$

whence $\tilde{u}=c \cos \theta$ and $\tilde{v}=-c \sin \theta$. Equation (25a) correspondingly requires that $\partial \theta / \partial \tilde{t}=0$, thereby, emphasizing that $\theta$ is stationary as seen by an observer in the moving frame. Equation (25c) immediately yields

$$
\frac{d^{2} \theta}{d \tilde{s}^{2}}+\left(c S^{1 / 3} \cos \theta-\frac{\mathcal{P}}{S^{1 / 3}}\right) \frac{d \theta}{d \tilde{s}}+c \sin \theta=0,
$$

which reduces to Eq. (18) of Ref. [7] by letting $\mathcal{P} \rightarrow A / 2$, $S^{1 / 3} \rightarrow \chi^{-1}, \tilde{s} \rightarrow 2 C_{f} s$, and $c \rightarrow\left(2 C_{f}\right)^{-1} c$. We seek finiteamplitude periodic solutions of Eq. (34) of the form

$$
\theta(\tilde{s})=\sum_{l=-\infty}^{+\infty} \Theta_{l} e^{i l q \tilde{s}}
$$

with $\Theta_{-l}=\Theta_{l}^{*}$ to ensure the reality of $\theta$, and with centerline wavelength $\widetilde{L}=2 \pi / q$. Accordingly, working to third order in $\theta$, Eq. (34) reduces to

$$
\begin{aligned}
{[1+} & \left.i q l\left(S^{1 / 3}-\frac{\mathcal{P}}{c S^{1 / 3}}\right)-\frac{q^{2} l^{2}}{c}\right] \Theta_{l} \\
& =\sum_{m, n=-\infty}^{+\infty}\left[\frac{1}{6}+\frac{1}{2} i q(l-m-n) S^{1 / 3}\right] \Theta_{l-m-n} \Theta_{m} \Theta_{n} .
\end{aligned}
$$

Equation (27) then implies the third-order sinuosity,

$$
S=1+\sum_{l=0}^{\infty}\left|\Theta_{l}\right|^{2}
$$

Equations (36) and (37) immediately yield the desired finite-amplitude solution. Linearizing these equations in the $l= \pm 1$ modes $\Theta_{ \pm 1}^{(1)} \equiv \mp i \epsilon / 2$ gives $c^{(0)}=q^{(0) 2}=\mathcal{P}$ and $S^{(0)}$ $=1$, in agreement with Eqs. (32) with $q=q_{c}$. Expanding in powers of the angle amplitude $\epsilon$ according to

$$
c=c^{(0)}+c^{(1)}+\cdots,
$$

$$
\begin{gathered}
q=q^{(0)}+q^{(1)}+\cdots, \\
S=S^{(0)}+S^{(1)}+\cdots, \\
\Theta_{l}=\Theta_{l}^{(1)}+\cdots,
\end{gathered}
$$

gives $c^{(1)}=q^{(1)}=S^{(1)}=\Theta_{l}^{(2)}=0$ to second order, and $c^{(2)}$ $=-\mathcal{P} \epsilon^{2} / 24, \quad q^{(2)}=-\mathcal{P}^{1 / 2} \epsilon^{2} / 12, \quad S^{(2)}=\epsilon^{2} / 4, \quad$ and $\quad \Theta_{ \pm 3}^{(3)}$ $=\left(\mathcal{P}^{1 / 2} \mp i / 3\right) \epsilon^{3} / 128$ to third order, whence

$$
\begin{gathered}
c=\mathcal{P}\left(1-\frac{\epsilon^{2}}{24}\right), \\
q=\mathcal{P}^{1 / 2}\left(1-\frac{\epsilon^{2}}{12}\right), \\
S=1+\frac{\epsilon^{2}}{4}, \\
\theta(\tilde{s})=\epsilon \sin (q \tilde{s})+\frac{\epsilon^{3}}{64}\left(\mathcal{P}^{1 / 2} \cos 3 q \tilde{s}+\frac{1}{3} \sin 3 q \tilde{s}\right) .
\end{gathered}
$$

Equations (39) agree with Eqs. (19)-(21) of Ref. [7], where Eq. (39d) is called the Kinoshita curve. Thus, we can recover the known finite-amplitude solution without resorting to a bend equation.

\section{TIME-DEPENDENT NONLINEAR MODAL ANALYSIS}

To study the time development of periodic meander patterns, we employ general time-dependent expansions

$$
\begin{aligned}
& \theta(\tilde{s}, \tilde{t})=\sum_{l=-\infty}^{+\infty} \theta_{l}(\tilde{t}) e^{i l q \tilde{s}}, \\
& \tilde{\kappa}(\tilde{s}, \tilde{t})=\sum_{l=-\infty}^{+\infty} \kappa_{l}(\tilde{t}) e^{i l q \tilde{s}}, \\
& \tilde{v}(\tilde{s}, \tilde{t})=\sum_{l=-\infty}^{+\infty} v_{l}(\tilde{t}) e^{i l q \tilde{s}},
\end{aligned}
$$

with time-dependent centerline wave number $q=2 \pi / \widetilde{L}$ and wavelength $\widetilde{L}$, time-independent cartesian wave number $q_{0}$ $=2 \pi / \widetilde{L}_{0}$ and wavelength $\widetilde{L}_{0}$, sinuosity $S=\widetilde{L} / \widetilde{L}_{0}=q_{0} / q$, and the reality conditions $\theta_{-l}=\theta_{l}^{*}, \tilde{\kappa}_{-l}=\tilde{\kappa}_{l}^{*}$, and $\tilde{v}_{-l}=\tilde{v}_{l}^{*}$. We take $\tilde{y}(0, \tilde{t})=\tilde{y}(\widetilde{L}, \tilde{t})$ to align the river axis with the $x$ axis, whence Eq. (26b) implies that $\theta_{0}=0$. Setting $\tilde{u}_{0}=\tilde{u}_{L}$ and substituting these expansions into Eqs. (25) gives

$$
\begin{gathered}
\kappa_{l}=-i l q \theta_{l}, \\
v_{l}=A_{l} \kappa_{l},
\end{gathered}
$$




$$
\frac{d S}{d \tilde{t}}=q^{2} S \sum_{l=-\infty}^{+\infty} l^{2} A_{l}\left|\theta_{l}\right|^{2}=2 q^{2} S \sum_{l=1}^{\infty} l^{2}\left|\theta_{l}\right|^{2} \operatorname{Re} A_{l},
$$

$$
\begin{aligned}
\frac{d \theta_{l}}{d \tilde{t}}= & \left(l^{2} q^{2} A_{l}+i l q \tilde{u}_{0}\right) \theta_{l}-q^{2} \\
& \times \sum_{\substack{m, n=-\infty \\
m \neq n}}^{+\infty} \frac{m n}{m-n} A_{n}\left[l \theta_{l}-(l+m-n) \theta_{l+m-n}\right] \theta_{-m} \theta_{n}
\end{aligned}
$$

where the coefficient,

$$
A_{l}=\frac{\mathcal{P}-i l q S^{1 / 3}}{1+i l q S^{1 / 3}} S^{-1 / 3}=\frac{\mathcal{P}-l^{2} q^{2} S^{2 / 3}}{1+l^{2} q^{2} S^{2 / 3}} S^{-1 / 3}-\frac{1+\mathcal{P}}{1+l^{2} q^{2} S^{2 / 3}} i l q,
$$

satisfies $A_{-l}=A_{l}^{*}$, and where we have taken care to include the time dependence of $q$ on the left side of Eq. (25a). The results of Sec. V follow by linearizing Eqs. (41) with $\theta_{1}$ $=\epsilon e^{-i \Omega t}$. The results of Sec. VI also follow from Eqs. (41) by writing $d \theta_{l} / d \tilde{t}=0, \quad \theta_{\mp 1}^{(1)}=\mp i \epsilon / 2$, and $\tilde{u}_{0}=\left.c \cos \theta\right|_{s=0}$ $=c \cos \left(\Sigma_{l=-\infty}^{+\infty} \theta_{l}\right)$, and by expanding in powers of $\epsilon$. Although Eqs. (36) and (37) describe steady periodic meander patterns valid only to third order in $\epsilon$, Eqs. (41) describe fully nonlinear time-dependent periodic meander patterns. In contrast with a previous analysis by Zolezzi [12], which includes only the modes $\theta_{1}, \theta_{3}$, and $\theta_{5}$, our Eqs. (41d) include all modes. Furthermore, we also introduce Eq. (41c), which governs the explicit evolution of $S$.

To study the time-dependent nonlinear dynamics governed by Eqs. (41), it is convenient to assign the $\tilde{s}=0$ point on the river to a bend apex, where

$$
\theta(0, \tilde{t})=\sum_{l=-\infty}^{+\infty} \theta_{l}(\tilde{t})=2 \sum_{l=1}^{\infty} \operatorname{Re} \theta_{l}=0,
$$

for all time, so that $\tilde{u}_{0} \equiv c$ is the time-dependent downstream migration rate of the apex. In contrast with the steady solutions of Sec. VI, other points along the periodic meander pattern, such as the $x$-axis crossings, do not generally migrate downstream with the same speed $c$.

For fixed $\mathcal{P}$ and fixed $q_{0}=q S$, Eqs. (41c), (41d), and (42) together determine the time-dependent sinuosity $S$, apex downstream migration rate $c=\tilde{u}_{0}$, and complex mode amplitudes $\theta_{l}$. Setting $\theta_{l}=0$ except for $l= \pm 1, \pm 2, \ldots, \pm N$ reduces Eqs. (41d) to $N$ complex equations for $l=1,2, \ldots, N$, with $\theta_{-l}=\theta_{l}^{*}$. Invoking forward differences, with integration time step $\Delta \tilde{t}$, to approximate the time derivatives in Eqs. (41c) and (41d), explicit numerical solutions for $\theta_{l}, S$, and $c$ are easily obtained.

For the initial condition $\theta_{l}=0$ except for $\theta_{ \pm 1}=\mp i \epsilon / 2$, which corresponds to the sine-generated curve $\theta(\tilde{s})$ $=\epsilon \sin q \tilde{s}$, Eqs. (41d) require growth of the higher-order odd modes $l= \pm 3, \pm 5$, etc., but fail to couple to any even modes with $l=0, \pm 2$, \pm 4 , etc. The cubic nonlinearity in Eqs. (41d) also fails to couple to any even modes when the initial condition includes higher-order odd modes, as noted previously by Seminara [11]. This can be seen from the structure of the cubic terms in Eqs. (41d), which require $\theta_{l}$ $=0$ for all even $l$ for all time if these modes vanish initially. If the initial condition includes even modes, our simulations show that these modes decay quickly to zero. A physical reason for this restriction to odd modes is still lacking.

To test the accuracy of results for different values of $N$, we compare values of $S=\widetilde{L} / \widetilde{L}_{0}$ obtained from Eq. (41c) with values obtained by integrating Eq. (27), with $\widetilde{L}=2 \pi / q$. For these tests, we used the sine-generated curve as the initial condition with $\epsilon=0.01$, and used $\mathcal{P}=5, q=q_{m}=1.20$, and $\Delta \widetilde{t}=0.001$. Allowing the bend amplitude to grow until the sinuosity reaches the value $S=7$, we found that values of $S$ determined by the two methods differ by at most $7 \%, 2 \%$, $0.5 \%$, and $0.2 \%$ for $N=3,5,7$, and 9 , respectively. Thus, the agreement between the two methods quickly improves with increasing $N$, and $N=9$ yields sufficiently accurate results.

\section{RESULTS AND CONCLUSIONS}

Of particular interest is the sinuosity $S_{c}$ at oxbow cutoff, when adjacent river bends meet, whereupon the river ceases to flow through a loop of the river called an oxbow lake. For periodic rivers, one oxbow lake is cut off for each wavelength of the river, and $S_{c}$ represents the maximum sinuosity of the river. Here we calculate $S_{c}$ for zero-width periodic rivers, that is, the value of $S$ when the centerlines of adjacent bends first meet each other. This value represents an upper limit on the cutoff sinuosity for finite-width periodic rivers, which cut off at smaller sinuosities, when the banks of adjacent bends (rather than their centerlines) meet each other, or when floods erode the narrow strips of land between adjacent bends.

To calculate $S_{c}$, we employ $\mathcal{P}=5, q=q_{m}=1.20$, and $\Delta \tilde{t}=0.001$ to integrate Eqs. (41c) and (41d), and then use Eqs. (26a) and (26b) to produce snapshots of the shapes of the river centerline at various times during the growth of the bend (see Fig. 3 of Ref. [13], for example). The cutoff time, sinuosity, and downstream apex migration rate for a particular value of $N$ follow when the centerlines of adjacent bends meet in such a snapshot. These values (symbols), together with their exact $N \rightarrow \infty$ limits $t^{\prime}=5.690, S=6.6945$, and $c$ $=-0.098971$ (dotted lines) are plotted in Fig. 6. Thus, at cutoff, the river is stretched to 6.6945 times the distance between its endpoints. The downstream apex migration rate $c$ at cutoff converges much more slowly than $t^{\prime}$ and $S$ because it is much more sensitive to the details of the shape of the river. The positive values $c=+0.450$ and $c=+0.0418$ for $N=1$ and $N=3$ (off the scale of Fig. 6) imply downstream migration, in contrast with the negative values for larger $N$, which imply upstream migration. Previous calculations by Seminara (Ref. [11]) for $N \leqslant 3$ fail to capture this upstream migration prior to cutoff.

Growth of the amplitude of long-wavelength bends occurs only for dimensionless centerline wave numbers $q<q_{c}$, 


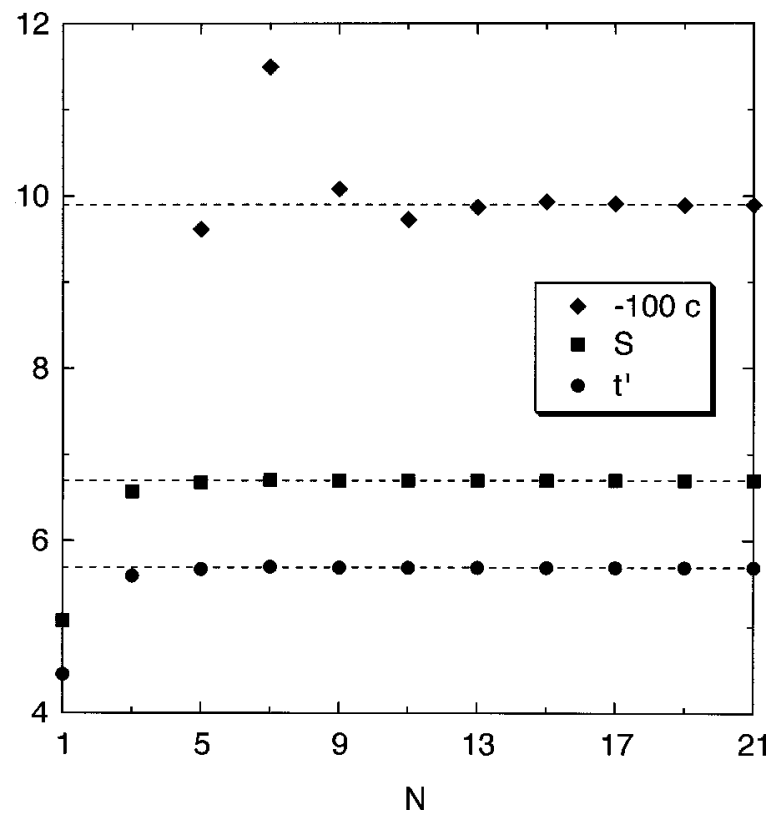

FIG. 6. Time (circles), sinuosity (squares), and downstream apex migration rate (diamonds) at oxbow cutoff predicted by truncating and integrating Eqs. (41c) and (41d), versus the truncation parameter $N$, together with exact results for these quantities (dashed lines). The negative values of $c$ indicate that bend apexes actually travel upstream at cutoff.

where $q=2 \pi / \tilde{L}$ involves the dimensionless centerline wavelength $\widetilde{L}=L / D_{0}$ measured along the river centerline, which equals or exceeds the usual dimensionless cartesian wavelength $\widetilde{L}_{0}=L_{0} / D_{0}=\widetilde{L} / S$ measured along a straight line. An upper limit on $q_{c}$ can be obtained by rewriting Eq. (41c) as

$$
\frac{d S}{d \tilde{t}}=-2 q^{2} S^{2 / 3} \sum_{l=1}^{\infty} \frac{l^{2} q^{2}-\mathcal{P} S^{-2 / 3}}{l^{2} q^{2}+S^{-2 / 3}} l^{2}\left|\theta_{l}\right|^{2} .
$$

Accordingly, if

$$
q>\frac{\mathcal{P}^{1 / 2}}{S^{1 / 3}}
$$

holds initially, then all modes make negative contributions to $d S / d t$ for all times because $q_{0}=q S$ is fixed. The sinuosity $S$ of such short-wavelength rivers must, therefore, decrease monotonically with time. This fully nonlinear result is valid for arbitrary finite-amplitude rivers of any shape, and supplies an upper limit $q_{c}=\mathcal{P}^{1 / 2} / S^{1 / 3}$ on the exact critical wave number. This limit exceeds the exact critical wave number by at most $10 \%$ over the full range of possible sinuosities [13]. In summary, an arbitrary periodic river whose centerline wave number satisfies Eq. (44) will always straighten with time, eventually becoming a straight line, regardless of the detailed initial shape of the river.

Remarkably, Eq. (44) follows simply by extending the exact linear growth condition to a sinuous finite-amplitude river. The linear condition (Sec. V) states that perturbations about a straight river decay in amplitude if their wavelengths satisfy $\lambda<2 \pi D_{0} / \mathcal{P}^{1 / 2}$ in conventional units, where $D_{0}$ $=H_{0} / 2 C_{f}$ is the decay length for a straight river. Extending this linear condition to a sinuous river with decay length $D$ $=H / 2 C_{f}=D_{0} S^{1 / 3}[\mathrm{Eq}$. (23)] gives the nonlinear growth condition

$$
\lambda<\frac{2 \pi}{\mathcal{P}^{1 / 2}} D
$$

where $\lambda$ is measured in conventional units along the river centerline. Equation (44) follows immediately as the corresponding dimensionless condition on $q=2 \pi D_{0} / \lambda$.

Perturbations of centerline wavelength $\lambda$ satisfying Eq. (45) decay with time. The critical wavelength scales with $D$, as might have been expected from the discussion in Sec. IV. Why, though, should this nonlinear condition follow as the simple extension of a linear condition valid only for small perturbations about a straight river? The answer lies in the linearity of the meandering equation, Eq. (25c), which determines the sinuosity-dependent phase lag between the velocity and curvature [compare Eq. (32d)],

$$
\delta=\tan ^{-1}\left[\frac{(1+\mathcal{P}) S^{-1 / 3} q}{\mathcal{P} S^{-2 / 3}-q^{2}}\right],
$$

which takes responsibility for the stability of individual modes. This phase lag follows from Eq. (25c) by setting $\tilde{\kappa}$ $=\kappa_{m} e^{i q \tilde{s}}, \tilde{v}=v_{m} e^{i q \tilde{s}}$, and $v_{m}=\rho e^{-i \delta} \kappa_{m}$. Just as for the linear problem, the phase-lag condition $\delta>\pi / 2$ leads directly to the stability condition, Eq. (44). For meander bends to grow with time, the normal velocity must point in the direction opposite the center of curvature of the channel. Since $\tilde{v}$ and $\tilde{\kappa}$ are, respectively, defined as positive for growth to the left and curvature to the right as seen by a river-bound observer facing downstream, $\tilde{v} \tilde{\kappa}>0$ implies growth and $\tilde{v} \tilde{\kappa}<0$ implies decay. For $\delta<\pi / 2, \tilde{v} \tilde{\kappa}>0$ for over half the wavelength, leading to net growth, whereas for $\delta>\pi / 2, \tilde{v} \tilde{\kappa}<0$ for over half the wavelength, leading to net decay. The stretching nonlinearity in the equation of motion (25a) governs the relative contributions of the individual modes, but not their stability. The meandering equation, Eq. (25c), takes full responsibility for the stability of individual modes.

In summary, we have used the equation of motion introduced by Seminara and co-workers $[11,12]$, the channel migration rate of Ikeda, Parker, and Sawai [3], and our own equation for the time-dependent river length to study the meandering of periodic rivers. This approach allows us to recover the known length scale $D$ for meandering and to introduce the associated time and velocity scales $T$ and $D / T$, which await experimental verification. An explicit equation governing the river sinuosity, which is derived from our river length equation, allows us to analytically determine the critical wavelength for nonlinear river meandering. This critical wavelength separates short-wavelength bends, which straighten with time, from long-wavelength bends, which grow. A general time-dependent nonlinear modal analysis for periodic rivers reveals that modes higher than the third mode 
are needed to describe upstream migration of bend apexes just before oxbow cutoff, and are important to accurate calculations of the time and sinuosity at cutoff.

Our physical approach yields a better understanding of the basic mechanisms of meandering. We attribute the decay of small-wavelength meander bends to Bernoulli shear caused by the cross-stream surface elevation gradient. We trace the origin of neutral meandering stability to the competition between Bernoulli shear and the secondary flow. We show that the length scale $D$ is the downstream distance required for cross-stream shear to recover from changes in the channel curvature, and that $D$, therefore, plays a key role in downstream migration of river bends.

\section{ACKNOWLEDGMENTS}

We gratefully acknowledge fruitful discussions and correspondence with Goodarz Amahdi, Ray Boswell, S. F. Edwards, Donald Gray, Peter Haff, Curtis Huffman, Hiroshi Ikeda, Alan Kerstein, Stephen Kite, Ray Lopez, Mark McKoy, Gary Parker, Jonathan Selinger, James Sethian, Jeffrey Skousen, Jeffrey Spooner, Tao Sun, Joseph Wilder, and the staff of the Earth Resources Observation Systems (EROS) Data Center.
[1] F. K. Lutgens and E. J. Tarbuck, Essentials of Geology (Prentice Hall, Englewood Cliffs, NJ, 1995), Chap. 9.

[2] Great Lakes Commission Staff, Dredging and the Great Lakes (Great Lakes Commission, Ann Arbor, MI, 1999), p. 6.

[3] S. Ikeda, G. Parker, and K. Sawai, J. Fluid Mech. 112, 363 (1981).

[4] H. Johannesson and G. Parker, in Water Resources Monograph No. 12: River Meandering, edited by S. Ikeda and G. Parker (American Geophysical Union, Washington DC, 1989), pp. 181-213

[5] G. Seminara and M. Tubino, J. Fluid Mech. 244, 257 (1992); P. Blondeaux and G. Seminara, ibid. 157, 449 (1985).

[6] G. Parker, K. Sawai, and S. Ikeda, J. Fluid Mech. 115, 303 (1982).

[7] G. Parker and E. Andrews, J. Fluid Mech. 162, 139 (1986).

[8] H. Johannesson and G. Parker, J. Hydraul. Eng. 115, 289 (1989).

[9] P. Meakin, T. Sun, T. Jossang, and K. Schwarz, Physica A 233, 606 (1996); T. Sun, P. Meakin, T. Jossang, and K. Schwarz, Water Resour. Res. 32, 2937 (1996).

[10] H. H. Stolum, Science 271, 1710 (1996); Phys. Rev. E 56, 6710 (1997); Geol. Soc. Am. Bull. 110, 1485 (1998).

[11] G. Seminara, in Nonlinear Dynamics and Pattern Formation in the Natural Environment, edited by A. Doelman and A. van Harten, Pitman Research Notes in Mathematics Series Vol. 335 (Longman, New York, 1995), pp. 269-294.

[12] G. Zolezzi, Ph.D. thesis, University of Genova, 1999.

[13] B. F. Edwards and D. H. Smith, Phys. Rev. E 63, R045304 (2001).
[14] L. B. Leopold and M. G. Wolman, Bull. Geol. Soc. Am. 71, 769 (1960).

[15] B. F. Edwards and D. H. Smith (unpublished).

[16] E. J. Hickin and G. C. Nanson, Geol. Soc. Am. Bull. 86, 487 (1975); G. C. Nanson and E. J. Hickin, ibid. 97, 497 (1986), and references contained therein.

[17] A. D. Howard and T. R. Knutson, Water Resour. Res. 20, 1659 (1984).

[18] T. B. Liverpool and S. F. Edwards, Phys. Rev. Lett. 75, 3016 (1995); T. B. Liverpool, R. C. Ball, and S. F. Edwards, Europhys. Lett. 30, 181 (1995).

[19] W. B. Langbein and L. B. Leopold, U. S. Geological Survey Professional Paper 422H, 1966 (unpublished).

[20] F. M. Henderson, Open Channel Flow (MacMillan, New York, 1966), Ch. 10.

[21] F. M. Henderson, Open Channel Flow (MacMillan, New York, 1966), Ch. 4.

[22] F. M. Henderson, Open Channel Flow (MacMillan, New York, 1966), Ch. 7.

[23] J. Zhou, H. H. Chang, and D. Stow, J. Hydraul. Eng. 146, 73 (1993).

[24] S. Ikeda and T. Nishimura, J. Hydraul. Eng. 112, 562 (1986).

[25] H. Kikkawa, S. Ikeda, and A. Kitagawa, J. Hydraul. Div., Am. Soc. Civ. Eng. 102, 1327 (1976).

[26] A. J. Odgaard, J. Hydraul. Div., Am. Soc. Civ. Eng. 107, 1677 (1981).

[27] G. Parker, P. Diplas, and J. Akiyama, J. Hydraul. Div., Am. Soc. Civ. Eng. 109, 1323 (1983). 